\title{
Trouser tear testing of thin anisotropic polymer films and laminates
}

\author{
Md Shafiqul Islam • Eskil Andreasson • \\ Sharon Kao-Walter
}

Received: 7 January 2019 / Accepted: 6 September 2019 / Published online: 17 September 2019

(C) The Author(s) 2019

\begin{abstract}
This research has investigated the essential work of fracture (EWF) from trouser tear test of polyethylene terephthalate (PET), low-density polyethylene (LDPE) films and their corresponding laminate using a convenient cyclic tear test method. Propagation of tear crack in these thermoplastics deflects from the initial crack path due to the material anisotropy. An improvement to a two-zone tear model for determining tear EWF was proposed for LDPE-like materials. Energy dissipation due to non-uniform bending of the trouser-legs was determined to be significant in EWF calculation of tearing and this was therefore considered in this study. To measure the tear EWF in laminates, contribution from delamination energy dissipation was accounted for.
\end{abstract}

Keywords Trouser tear test - Essential work of fracture $\cdot$ Flexible laminate Crack path deviation . Delamination

\footnotetext{
M. S. Islam $(\varangle) \cdot$ E. Andreasson · S. Kao-Walter

Dept. of Mech. Eng., Blekinge Institute of Technology,

37179 Karlskrona, Sweden

e-mail: shafiqul.islam@bth.se

E. Andreasson

Tetra Pak, 22355 Lund, Sweden

e-mail: eskil.andreasson@tetrapak.com

S. Kao-Walter

Fac. of Mech. \& El. Eng., Shanghai Polytechnic Univ.,

Shanghai 201209, China

e-mail: sharon.kao-walter@bth.se
}

\section{Introduction}

Polymers films of low-density polyethylene (LDPE) and polyethylene terephthalate (PET) are widely used in packaging industries, and tearing is a common method of package-opening. Although there are many existing in-plane mode I studies on single layer as well as laminates of these thin polymer films (Kao-Walter 2004; Kao-Walter et al. 2006; Andreasson et al. 2014; Zhang et al. 2016), not many out-of-plane investigations could be found in the literature (Kim and KargerKocsis 2004; Bjerkén et al. 2006; Kao-Walter et al. 2009, 2011; Martínez et al. 2010; Andreasson et al. 2013).

The essential work of fracture (EWF) of tear in thin polymer films has increased popularity to characterize out-of-plane shear fracture toughness (Wong et al. 2003). The two-leg trouser tear test which was first used by Rivlin and Thomas (1953) with rubber, rapidly became a preferred test method for tear testing of thin sheets and films. 'Trouser tear test' will be referred simply as 'tear test' later in this article. One of the challenges is that, when highly extensible materials experience tearing, it is hard to separate the plastic work done in the legs from the plastic work done at the vicinity of the crack. The total fracture energy from a tear test can be separated into geometry dependent (non-essential work) and geometry independent (essential work) contributions to characterize EWF. For energy separation in a tear test, a two-zone model was proposed by Wong et al. (2003). The authors showed that the plastic 
zone width increases with progress of tearing up to a certain length (zone one) from the initial crack tip; and for any further tearing, the plastic zone width remains constant (zone two). This research was extended by Kim and Karger-Kocsis (2004), who included a third zone that divides 'zone one' proposed by Wong et al. (2003) into two separate zones, wherein the new 'zone one' considers the crack tip deformation prior to any crack propagation during a tear test. A two-zone model was selected as the foundation of the current study, and additional observations were incorporated, as discussed in Sect. 4. All the earlier studies used multiple tear tests for tear EWF calculation, whereas, the current study showed that a single cyclic tear test can be sufficient.

During a tear test, the specimen legs bends plastically close to the crack tip and along the leg width in some cases. Dissipation of energy from the work done by plastic bending and straightening of trouser-legs during tearing is non-essential work of fracture. It was considered for EWF calculation by Mai and Cotterell (1984) for metal with constant curvature of leg bending. Kim and Karger-Kocsis (2004) later reported that dissipation of energy due to plastic bending and straightening is negligible for polymers; they did not report any measurement on this. However, for PET, LDPE, and their laminate, the bending was observed to vary along the width of the specimen leg. The work of plastic dissipation from this non-uniform bending was considered as presented in Sect. 3, and its magnitude was determined to be significant to EWF as shown in Sect. 4.

Studying the tearing of thin polymer laminates become involved as the films in the laminate respond differently under load compared to individual layer; this has been investigated by several authors (Bjerkén et al. 2006; Andreasson et al. 2014; Zhang et al. 2016; Kao-Walter et al. 2006, 2009, 2011; Islam et al. 2016). Kao-Walter et al. (2011) investigated the LDPE-PET laminate under tearing and observed delamination in the interface which increased along with tear crack propagation. This study has also investigated the significance of delamination in a laminate while calculating laminate EWF.

Further, both LDPE and PET are anisotropic which resulted in deviation of the tearing crack from its initial path as it propagates. Mode mixing is another challenge. Wong et al. (2003) described mode III tearing EWF to be very similar to that of mode I and attributed this to the fact that mode III tearing at the crack tip becomes a mixture of mode I and mode III due to high local deformation (Kim and Karger-Kocsis 2004; Mai and Cotterell 1984). Bárány studied EWF of PET for mode I and mode III and found similar correlation between them (Bárány et al. 2005).

This article presents a new cyclic tear test method, proposes tear EWF calculation method for laminates and considers non-uniform bending and delamination in the trouser-legs as non-essential work of fracture. The effect of material anisotropy was also checked. The article is organized as follows: Experiments on standard tear test and its extension to cyclic tear teat is presented in Sect. 2. Section 3 describes the application of a existing plastic energy dissipation (nonessential work of fracture) theory to non-uniform bending of trouser-legs. EWF of tearing was calculated for thin LDPE, PET films and their laminate in Sect. 4. Section 5 presents some scanning electron microscope (SEM) observations of fracture surface and delamination of the tested materials. Results are discussed in Sect. 6 and the paper ends with some conclusions.

\section{Experiments}

A laminate of LDPE-PET film was examined in this study. The material was supplied by a packaging industry and is a constituent of liquid food packaging. The LDPE layer was separated from the PET film in the laminate manually in the laboratory to perform single layer tests. Specimens were cut from a roll of film as shown in Fig. 1a. A sharp surgical blade was used to cut the specimens and the pre-cracks. All tests were performed using an MTS Qtest universal tensile testing machine. The films to be tested were kept at a controlled laboratory environment with a temperature of $23^{\circ} \mathrm{C}$ and 50 $\%$ humidity for at least $24 \mathrm{~h}$ before specimen preparation. The tests were displacement-controlled. For any experimental results presented, at least three tests were performed.

Cyclic trouser tear tests were utilized to determine tear EWF in single layers and in the laminate. To complement the calculation of tear EWF and to find a relation between tear and mode I fracture, additional tensile tests were performed on the continuum and center crack specimens of the same materials.

LDPE, PET and LDPE-PET laminate are anisotropic. Tear and tensile tests were performed in five different material orientations as depicted in Fig. 1a for check- 
Fig. 1 a Material orientation for test samples of the anisotropic films and b studied specimen geometry

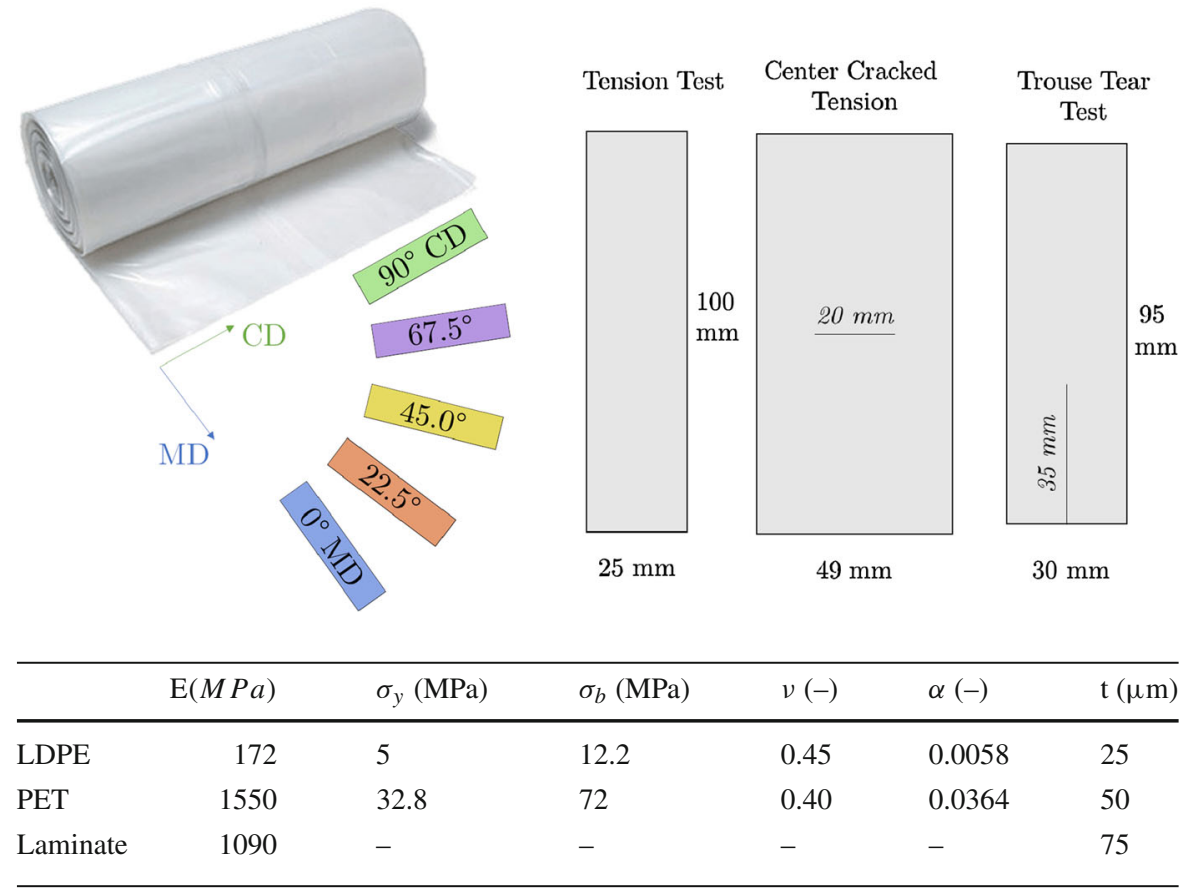

Table 1 Comparison of mechanical and geometric properties of the tested materials in MD

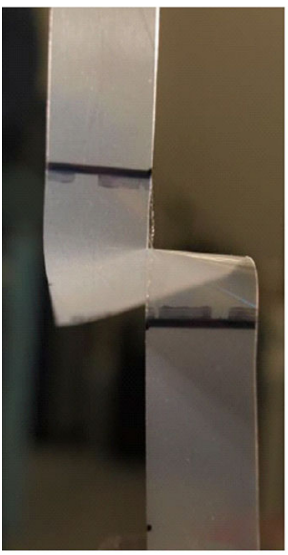

(a)

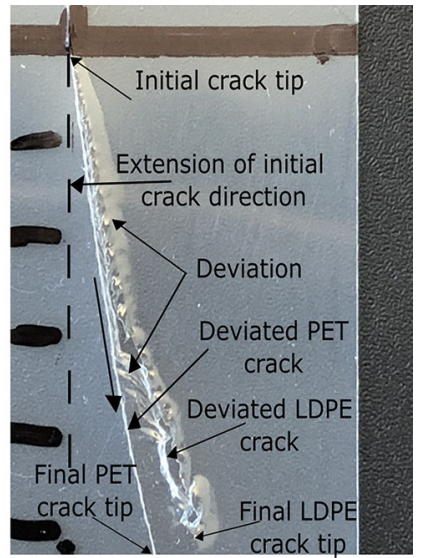

(b)
A standard two-leg trouser tear test method (Standard 1993) was adopted to check anisotropy in the studied polymers under tearing. The dimensions for the tear specimen is shown in Fig. 1b. The test was performed until the crosshead moved $20 \mathrm{~mm}$ to produce a $10 \mathrm{~mm}$ crack propagation. Influence of material anisotropy was observed on the tear peak load response, the deviation of the tear propagation path, and delamination due to tear. Tear crack deviation and delamination can be observed in the post-test LDPE-PET laminate in MD (Fig. 2b). Figure 3 shows tear force response at different orientations. The Laminate tear force exhibited
Fig. 2 Tearing of films: a a laminate under trouser tear test and b a post-test laminate specimen

larger anisotropy compared to the individual layers. A latter portion of this article explains the result through the crack deviation and delamination in the laminate.

Tear crack propagation deviated significantly from the initial direction for both laminate and PET (Figs. 4a and 5) but was within $1^{\circ}$ to $2^{\circ}$ for LDPE. These deviations were measured and presented in Table 2 along with the delamination area in the laminate caused dur- 
Fig. 3 Force displacement response of tearing a PET, $\mathbf{b}$ LDPE and $\mathbf{c}$ laminate. (For explanations of the colours in these figure legends, please refer to the web version of this article)

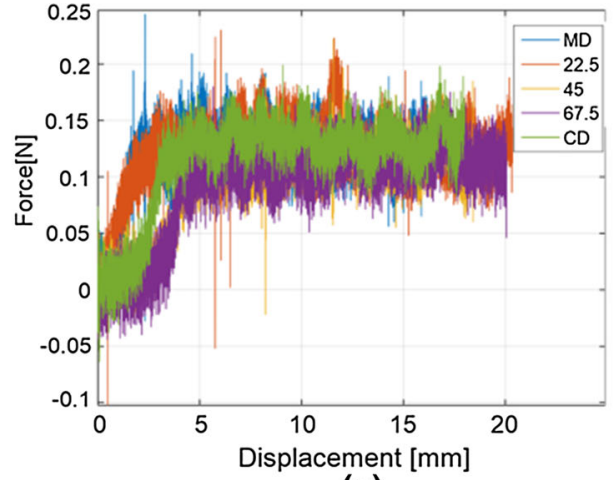

(a)

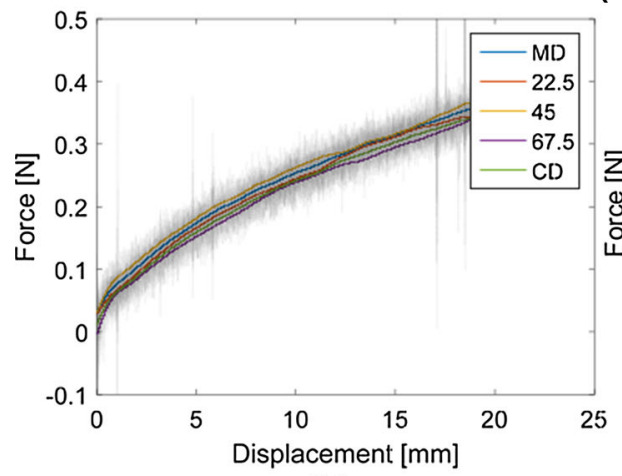

(b)

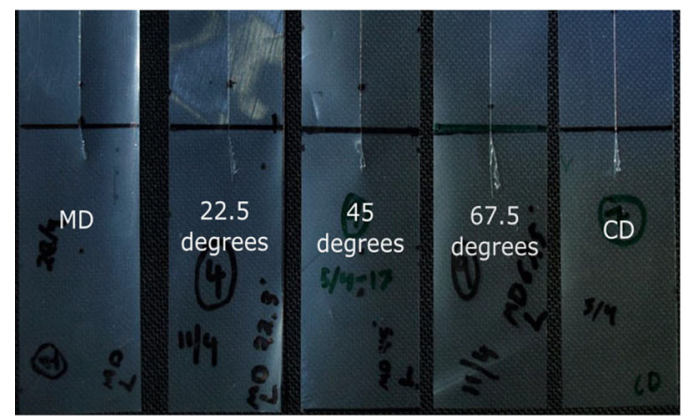

(a)

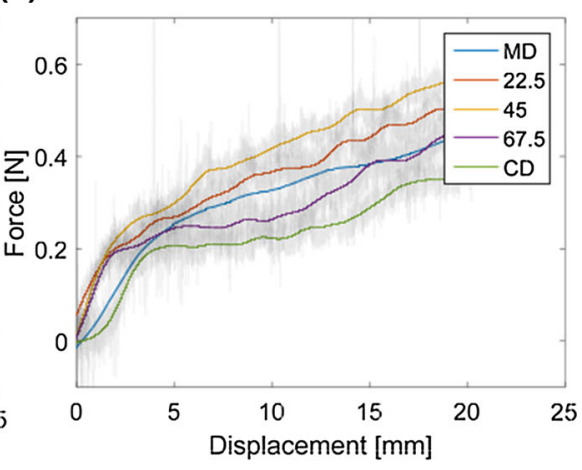

(c)
Fig. 4 a Crack deviation during tearing of laminate; b delamination during tearing of laminate
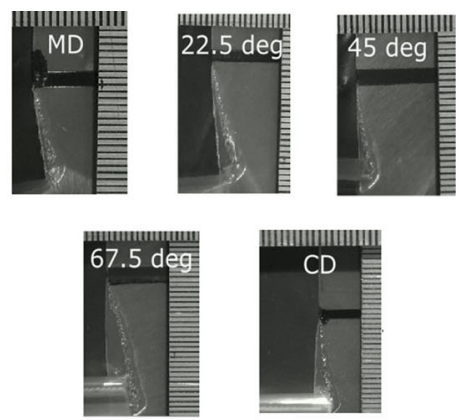

(b) ing the tearing. The area of delamination was measured based on the photographs (Fig. 4b) using a desktop application 'plotdigitizer' (JA 2010).

\subsection{Cyclic trouser tear test}

For EWF calculation, the specimens were torn only in MD to a larger extent through five incremental loading and unloading cycles. Figure 6 illustrates this incremental tearing through loading and unloading. Noticeably, the crack propagation can be expected to be nearly

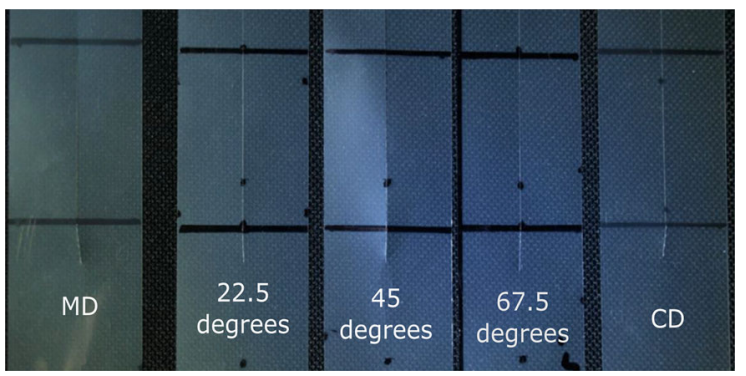

Fig. 5 Crack deviation during tearing of PET 
Table 2 Crack deviation in PET and laminate together with delamination due to tearing in degrees (small crack deviation of LDPE was disregarded)

\begin{tabular}{llllll}
\hline & MD & $22.5^{\circ}$ & $45^{\circ}$ & $67.5^{\circ}$ & CD \\
\hline Crack angle-PET & 12 & $2-4$ & 1 & $-(3-4)$ & -6 \\
Crack angle-laminate & $7-8$ & $6-7$ & 1 & $-(1-1)$ & $-(4-6)$ \\
Delaminated area $\left(\mathrm{mm}^{2}\right)$ & 6.30 & 5.32 & 3.30 & 5.572 & 7.66 \\
\hline
\end{tabular}

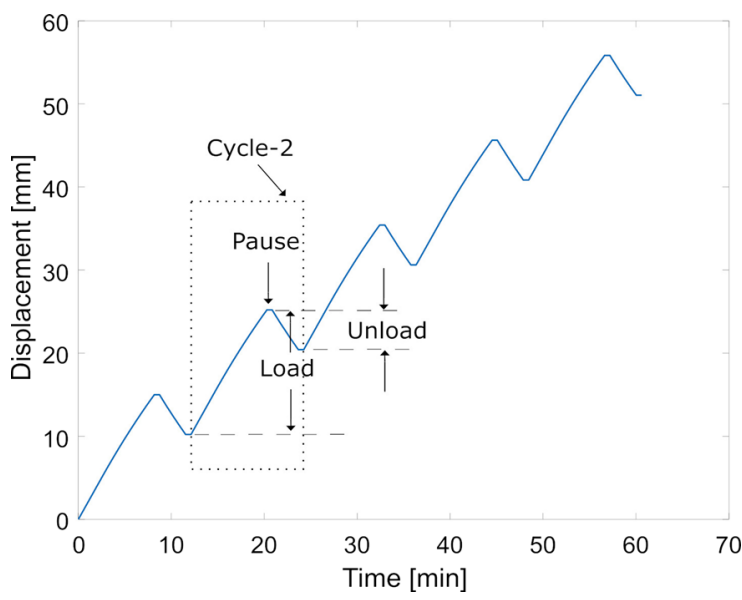

Fig. 6 Cyclic test method for incremental tearing

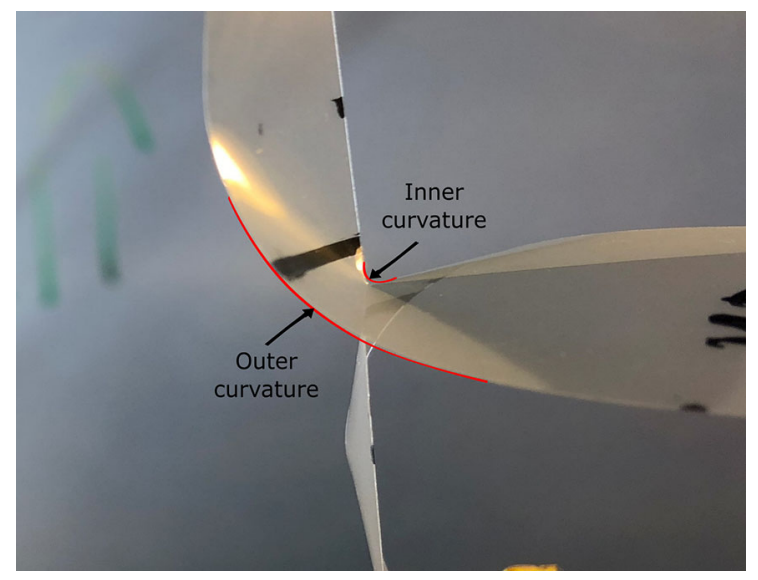

Fig. 7 Side view of trouser tear specimen, measuring the inner and outer tear bending curvature from images

\section{Bending dissipation of tear}

During tearing, the specimen legs endured beam-like bending and straightening, which may result in plastic energy dissipation. With the advance of tear precrack, the maximum bending of a trouser-leg changes position. At steady-state tearing, this change in position of the maximum curvature is equal to the growth in pre-crack $(d a)$. For energy dissipation during bending $\left(d U_{d b}\right)$ at maximum curvature, the bending energy release rate can be written as,

$G_{d b}=\frac{d U_{d b}}{d a}$

Kinloch et al. (1994) presented mathematical expressions for this bending dissipation for a bi-linear isotropic hardening material as a function of normalized curvature $\left(k_{0}\right)$ of bending (Kinloch et al. 1994). Depending on the maximum $k_{0}$ of the beam in a loading history, three probable cases may arise (Kinloch et al. 1994),

Case 1: For $0<k_{0}<1$, bending involves elastic loading and elastic unloading with no plasticity. LDPE in this study. 


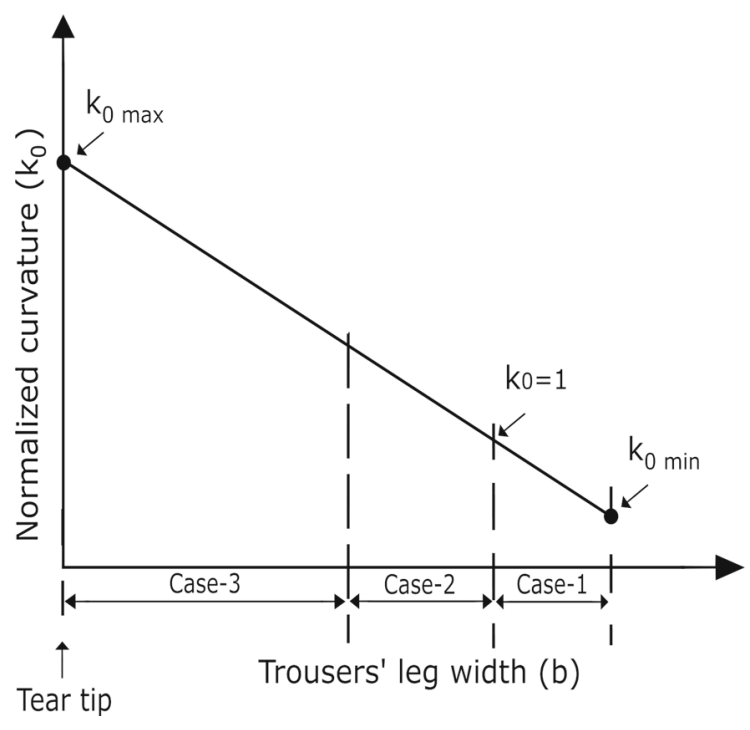

Fig. 8 Dividing the trouser-leg's width (b) based on level of plastic loading

Case 2: For $1<k_{0}<2$, bending involves elasticplastic loading and elastic unloading, but no reverse plasticity.

Case 3: For $k_{0}>2$, elastic-plastic loading and reverse plastic deformation are involved.

Tensile responses of the materials shown in Appendix A indicated that the hardening could be assumed bilinear for both materials in MD orientation. Furthermore, if isotropic hardening is assumed, then expressions by Kinloch et al. (1994) could be directly adopted to measure dissipation of plastic bending and unbending in the trouser test if bending of the legs was uniform. Instead, bending is non-uniform (Fig. 7), and it is highly probable that along the width $(b)$ of the trouser-legs bending, the material will experience all three possible cases of plastic energy dissipation with gradual changes in curvature, from inner to outer curvature (Fig. 8).

According to Fig. 7, the inner curvature $k_{\max }$ and outer curvature $k_{\min }$ were measured from the tear test image using the application plotdigitizer. It was further assumed that for a small increase in torn ligament length $\left(l_{a}\right)$, the curvatures remain same at steady state. The inner and the outer curvatures were then normalized using the equation below:

$k_{0 \max }=\frac{k_{\max }}{k_{1}} ; k_{0 \min }=\frac{k_{\min }}{k_{1}}$

$k_{1}=\frac{2 \varepsilon_{y}}{t}$
Here, $\varepsilon_{y}$ is the initial yield strain and $t$ is trouser-leg's thickness. Assuming a linear change of curvature along the width of the legs, the normalized curvature can be expressed as a function of width, $b$ (Eq. 4). The leg width can be divided into three zones as illustrated in Fig. 8.

$k_{0}(b)=k_{0 \max }-\frac{b}{b_{\max }}\left(k_{0} \max -k_{0 \min }\right)$

Analytical beam model for plastic energy dissipation due to bending used by citekinloch1994 takes the following form for non-uniform bending:

Case 1: For $b<\frac{b_{\max }\left(k_{0} \max -1\right)}{k_{0} \max -k_{0} \min }$; no plastic dissipation.

$G_{d b 1}=0$

Case 2: For $\frac{b_{\max }\left(k_{0} \max -1\right)}{k_{0} \max -k_{0} \min }<b<\frac{b_{\max }\left[k_{0} \max -\frac{2(1-\alpha)}{(1-2 \alpha)}\right]}{k_{0} \max -k_{0} \min }$

$G_{d b 2}=G_{\max }^{e}\left[(1-\alpha) \frac{k_{0}(b)^{2}}{3}+\frac{2(1-\alpha)^{2}}{3 k_{0}(b)}-1\right]$

Case 3: For $b>\frac{b_{\max }\left[k_{0 \max }-\frac{2(1-\alpha)}{(1-2 \alpha)}\right]}{k_{0} \max -k_{0} \min }$

$$
\begin{aligned}
G_{d b 3}= & G_{\max }^{e}\left[\frac{4}{3} \alpha(1-\alpha)^{2} k_{0}(b)^{2}\right. \\
& \left.+2(1-\alpha)^{2}(1-2 \alpha) k_{0}(b)\right] \\
& +G_{\max }^{e}\left[\frac{4(1-\alpha)\left[1+4(1-\alpha)^{3}\right]}{3(1-\alpha) k_{0}(b)}\right. \\
& \left.-2(1-\alpha)\left[1+4(1-\alpha)^{2}\right]\right]
\end{aligned}
$$

Here, $G_{\text {max }}^{e}$ is the maximum elastic energy in the specimen leg (for unit width, per unit crack propagation).

$G_{\max }^{e}=\frac{1}{2} E \varepsilon_{y}^{2} t$

Finally, bending dissipation per unit ligament length $\left(l_{a}\right)$,

$\frac{W_{d b}}{l_{a}}=\int_{b_{2} \min }^{b_{2} \max } G_{d b 2} d b+\int_{b_{3 \min }}^{b_{\max }} G_{d b 3} d b$ 
Here, $l_{a}$ is equivalent to tear crack length. This dissipation is non-essential work of fracture.

\section{Essential work of fracture}

As discussed previously, a two-zone model (Wong et al. 2003) was adopted for tear EWF calculation. In this model, total work of fracture for tear is calculated as follows (Wong et al. 2003):

$W_{T F}=W_{T E}+W_{T P}=w_{T e} l_{a} t+w_{T P} S_{p a} t$

$W_{T F}$ is total work, $W_{T E}$ is total essential work and $W_{T P}$ is total plastic work of tear fracture. Further, $w_{T e}$ is specific essential work and $w_{T P}$ is specific plastic work of tear fracture. Here, tear crack propagation length is referred to as ligament length $\left(l_{a}\right)$. Plasticized area $\left(S_{p a}\right)$ is the area of plastic zone near the crack propagation path (Fig. 9a, b). Some additional observations and considerations were incorporated into this model and are presented next.

\subsection{Separation of plastic dissipation from bending}

Plastic dissipation due to bending $W_{d b}$ (Eq. 9) in the trouser-legs was demonstrated to be significant in the current case. When $W_{d b}$ is accounted for, the expression in Eq. 10 takes the form as follows:

$$
\begin{array}{r}
W_{T F}-W_{d b}=W_{T E}+W_{T P} \\
=w_{T e} l_{a} t+w_{T P} S_{p a} t
\end{array}
$$

$W_{d b}$ can be calculated from experimental observations based on Eq. 9 as described in Sect. 3.

\subsection{Evaluation of EWF from only Zone I}

During a tear test, a plastic zone is developed close to the propagated crack. The width $(h)$ of the plastic zone (also called ligament width) increases as the crack tip progresses in a certain manner depending on the mechanical and geometric properties of the material. This is illustrated in the schematic of Fig. 9a. This plastic zone is visible in a teared LDPE specimen as wrinkles of increasing width (Fig. 9b) and as a thin white zone close to fracture surface for PET teared

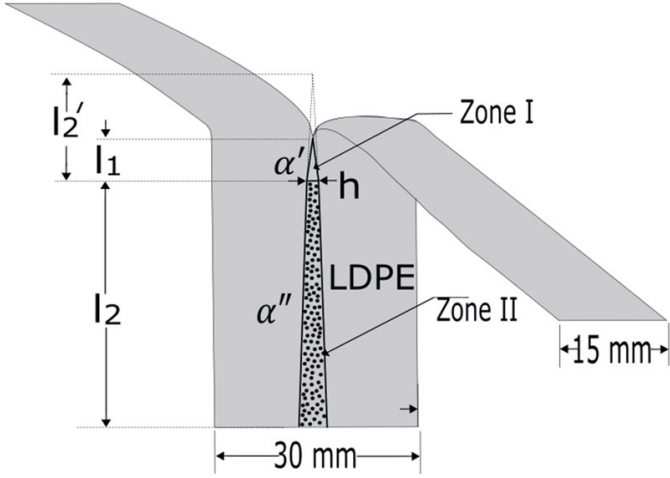

(a)

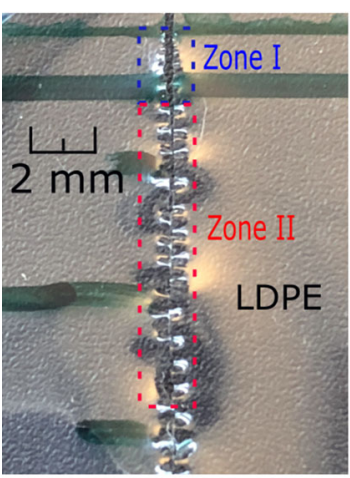

(b)

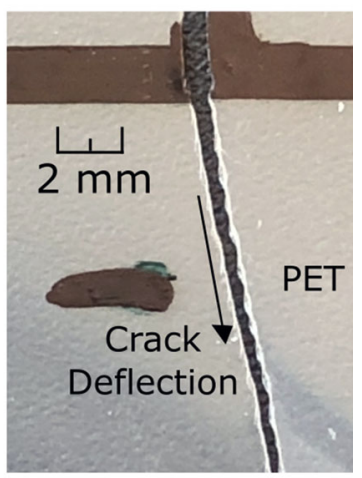

(c)
Fig. 9 Zones in a tear test. a Schematic for post-tear LDPE where $l_{a}=l_{1}+l_{2}$, b post-tear LDPE, c post-tear PET

specimen (Fig. 9c). Zone I of a post-tear specimen is the small zone ahead of initial crack tip where plastic zone width $(h)$ increases more rapidly. Observation of post-test tear specimens (Fig. 9c) indicates that the plastic zone area $\left(S_{p a}\right)$ for PET (for the tested thickness) was small and barely spread from the fracture surface. The subsequent SEM study made similar observations. However, LDPE plastic zone width increased faster in zone I (Fig. 9b). In zone II (Fig. 9b), the plastic zone width increased slowly and steadily with the increasing ligament length. The triangularly shaped plastic area in zone I (Fig. 9b) can be calculated as $S_{p a}=\alpha^{\prime} l_{a}{ }^{2}$. The plastic area multiplier, $\alpha^{\prime}$, as presented by Wong et al. (2003) is the slope of the ligament width outer boundary with respect to pre-crack path in zone I (Fig. 9a). Therefore, Eq. 11 can be written as follows:

$\frac{W_{T F}-W_{d b}}{l_{a} t}=w_{T e}+w_{T P} \alpha^{\prime} l_{a}$ 
The left side of Eq. 12 can be calculated based on experimental measurements. If several experiments can be performed within the zone I ligament length $\left(l_{a}\right)$, Eq. 12 can effectively be used to extrapolate the expression for zero ligament length and quantify the specific essential work of fracture $\left(w_{T e}\right)$.

\subsection{Evaluation of EWF from only zone II}

For PET, if contribution from the very small zone I is ignored, the model in Eq. 12 could be directly used for evaluation of EWF using zone II (zone II is treated as zone I). However, in a post-tear LDPE specimen, $l_{a}$ in zone I is small but significant and zone II is present. As illustrated in Fig. 9a, b, LDPE had a zone I (length $l_{1}$ ) with a sharp increase in ligament width $(h)$ (slope $\alpha^{\prime}$ ), followed by zone II (length $l_{2}$ ), in which width of the ligament $(h)$ increases comparatively slower (slope $\left.\alpha^{\prime \prime}\right)$. As in Fig. 9a, it is possible to extend zone II backwards to achieve zero ligament width at a distance of $l_{2}^{\prime}$ from the beginning of zone II. Further, total work of fracture can be partitioned as zone I and zone II work of fracture. The total work of fracture in zone I $\left(W_{T F-I}\right)$ can be experimentally quantified using Eq. 12. It is then possible to plot a relation between $W_{T F-I I}=W_{T F}-W_{d b}-W_{T F-I}$ and $l_{2}=l_{a}-l_{1}$. Figure 9a implies that specific plastic work of fracture $\left(w_{T P}\right)$ is zero at $l_{2}=-l_{2}^{\prime}$. Hence according to Eq. 14 , extrapolating this curve to $l_{2}=-l_{2}^{\prime}\left(l_{a}=l_{1}-l_{2}^{\prime}\right)$ provides the specific essential work of fracture.

$$
\begin{aligned}
W_{T F-I I}-W_{d b} & =W_{T E}+W_{T P} \\
& =w_{T e} l_{2} t+w_{T P} S_{p a} t \\
\frac{W_{T F-I I}-W_{d b}}{l_{2} t} & =w_{T e}+w_{T P}\left(h+2 \alpha^{\prime \prime} l_{2}\right)
\end{aligned}
$$

The length of $l_{2}^{\prime}$ can be calculated by measuring the zone II slope $\left(\alpha^{\prime \prime}\right)$ and maximum zone I width $(h)$,

$l_{2}^{\prime}=\frac{h}{2 \alpha^{\prime \prime}}$

At $l_{2}=-l_{2}^{\prime}$, the contribution from non-essential plastic dissipation $w_{T P}$ becomes zero.

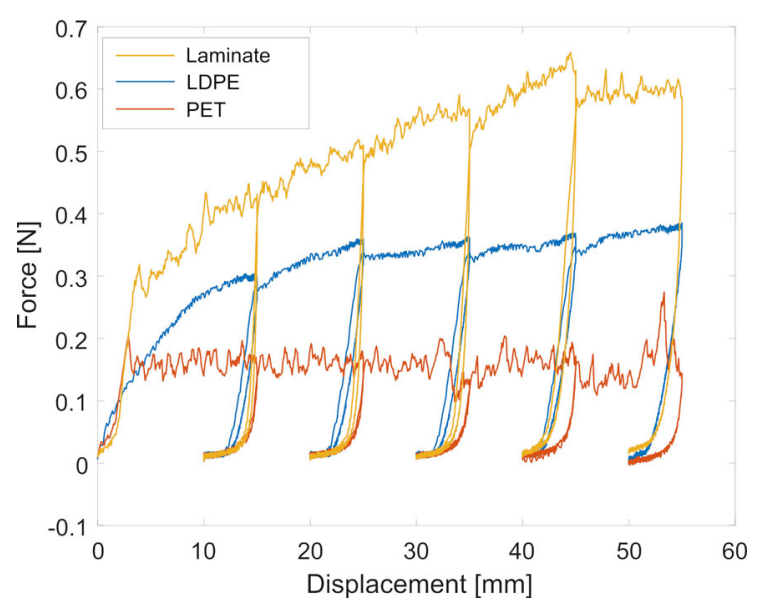

Fig. 10 Cyclic loading-unloading test response for tear; Laminate consists of LDPE and PET layers. (For explanations of the colours in this figure, please refer to the web version of this article)

\subsection{EWF in laminates}

In the laminate, as in Fig. 2b, PET layer crack deviates; however, it does so less than as a single layer (Table 2). The LDPE layer in the laminate follows a common deflected crack path with PET as long as the delamination is small near to initial crack tip (Fig. 2b). Since the PET layer is significantly stiffer and thicker than LDPE, it controls the laminate crack deviation. At the same time, since LDPE is more isotropic, this restrains the deviation of the laminate crack and results in less PET crack deviation in laminate than stand-alone tear at the beginning for tear. With increasing crack propagation, delamination increases, and the LDPE crack path deviates from that of PET. Importantly, the LDPE crack eventually deviates at least as much as PET or more because of the constraint that the stiffer PET layer places in softer LDPE in a laminate. This behaviour increases the amount of delamination with crack propagation and results in smaller crack propagation in LDPE than PET (Fig. 2b). For convenience of laminate EWF calculation, the propagated crack length up to the common crack path of LDPE and PET was assumed. The non-essential energy dissipation due to delamination $\left(W_{d e l}\right)$ must also be considered in the calculation and included in Eq. 16. 
Table 3 Specific work of PET fracture calculation for different cycles using Eq. 12

\begin{tabular}{lllll}
\hline PET (MD) & Ligament length $\left(l_{a}\right)[\mathrm{mm}]$ & $\begin{array}{l}\text { Total work of fracture } \\
\left(W_{T F}\right)[\mathrm{N}-\mathrm{mm}]\end{array}$ & $\begin{array}{l}\text { Bending dissipation }\left(W_{d b}\right) \\
{[\mathrm{N}-\mathrm{mm}]}\end{array}$ & $\begin{array}{l}\text { Specific total work of frac- } \\
\text { ture } w_{T e}+w_{T P} \alpha^{\prime} l_{a}[\mathrm{~N} / \mathrm{mm}]\end{array}$ \\
\hline Cycle-1 & 6.80 & 2.04 & 0.28 & 5.20 \\
Cycle-2 & 11.30 & 3.66 & 0.28 & 5.49 \\
Cycle-3 & 15.40 & 5.24 & 0.29 & 5.71 \\
Cycle-4 & 20.00 & 6.84 & 0.28 & 5.72 \\
Cycle-5 & 24.40 & 8.41 & 0.29 & 5.75 \\
\hline
\end{tabular}

Table 4 Specific work of LDPE fracture calculation for different cycles using Eq. 14

\begin{tabular}{|c|c|c|c|c|}
\hline LDPE (MD) & $\begin{array}{l}\text { Ligament length in zone II } \\
\left(l_{2}\right)[\mathrm{mm}]\end{array}$ & $\begin{array}{l}\text { Total work of fracture in } \\
\text { zone II }\left(\left(W_{T F}-W_{T F-I}\right)\right) \\
\text { [N-mm] }\end{array}$ & $\begin{array}{l}\text { Bending dissipation }\left(W_{d b}\right) \\
{[\mathrm{N}-\mathrm{mm}]}\end{array}$ & $\begin{array}{l}\text { Specific total work of frac- } \\
\text { ture in zone II } w_{T e}+ \\
w_{T P}\left(h+\alpha^{\prime \prime} l_{2}\right)[\mathrm{N} / \mathrm{mm}]\end{array}$ \\
\hline Cycle-1 & 4.80 & 2.51 & 0.10 & 20.11 \\
\hline Cycle-2 & 9.50 & 5.78 & 0.10 & 23.51 \\
\hline Cycle-3 & 14.00 & 9.36 & 0.10 & 25.88 \\
\hline Cycle-4 & 18.50 & 12.99 & 0.12 & 27.22 \\
\hline Cycle-5 & 22.90 & 16.78 & 0.13 & 28.44 \\
\hline
\end{tabular}

$W_{T F-I}=0.3144[\mathrm{~N}-\mathrm{mm}] ; \mathrm{h}=0.7 \mathrm{~mm} \alpha^{\prime \prime}=0.034$ and $l_{2}^{\prime}=10.3$

Table 5 Specific work of laminate fracture calculation for different cycles

\begin{tabular}{|c|c|c|c|c|c|}
\hline Laminate (MD) & $\begin{array}{l}\text { Ligament length } \\
\left(l_{a}\right)[\mathrm{mm}]\end{array}$ & $\begin{array}{l}\text { Total work of } \\
\text { fracture }\left(W_{T F}\right) \\
{[\mathrm{N}-\mathrm{mm}]}\end{array}$ & $\begin{array}{l}\text { Bending dissipa- } \\
\text { tion }\left(W_{d b}\right) \quad[\mathrm{N}- \\
\mathrm{mm}]\end{array}$ & $\begin{array}{l}\text { Delamination } \\
\text { dissipation } \\
W_{d e l}=w_{d e l} S_{d e l} \\
{[\mathrm{~N}-\mathrm{mm}]}\end{array}$ & $\begin{array}{l}\text { Specific total } \\
\text { work of fracture } \\
w_{T e}+w_{T P} \alpha^{\prime} l_{a} \\
{[\mathrm{~N} / \mathrm{mm}]}\end{array}$ \\
\hline Cycle-1 & 6.60 & 4.32 & 0.97 & 0.0179 & 6.63 \\
\hline Cycle-2 & 11.5 & 9.05 & 1.06 & 0.04 & 8.07 \\
\hline Cycle-3 & 15.50 & 14.52 & 1.18 & 0.06 & 9.62 \\
\hline Cycle-4 & 20.20 & 20.41 & 1.15 & 0.08 & 10.45 \\
\hline Cycle-5 & 24.80 & 26.64 & 1.21 & 0.15 & 11.13 \\
\hline
\end{tabular}

$$
\begin{aligned}
W_{T F}-W_{d b}-W_{d e l} & =W_{T E}+W_{T P} \\
& =w_{T e} l_{a} t+w_{T P} S_{p a} t \\
W_{d e l} & =w_{d e l} S_{d e l}
\end{aligned}
$$

The area of delamination $\left(S_{d e l}\right)$ can be measured from the post-tear specimen image, and $w_{d e l}$ can be calculated from peel tests as in Appendix C.

\subsection{Calculation of EWF from cyclic tear test}

Total work of fracture for different ligament lengths (crack propagation) can be quantified through a single cyclic tear test with loading to a certain ligament length of tearing and unloading to zero reaction force. Then subsequently re-load for tearing to a new ligament length greater than the first cycle and unload again. This cyclic loading and unloading described in Sect. 2 can be performed an arbitrary number of times. Figure 6 describes the test method. For any arbitrary length of tearing, the area under the load-unload curve provides the total work of fracture due to tear; additionally, if the tearing length is known, this can be used to calculate the specific total work of fracture using Eq. 12 or Eq. 14 for a single layer and Eq. 16 for laminate. The area under the curve for loading in cycle 1 is regarded as $A_{L-c 1}$ and as $A_{U-c 1}$ for unloading after crack prop- 
agation of length $l_{a 1}$. Specific total work of fracture for the tear in first cycle is as follows:

$w_{T F 1}=\frac{W_{T F 1}}{l_{a 1} t}=\frac{A_{L-c 1}-A_{U-c 1}}{l_{a 1} t}$

The loading for the second cycle can be considered to follow the same load-displacement path, initially, as for first cycle (Fig. 10) and eventually tear to a new ligament length $l_{a 2}$. Specific total work of fracture for the tear from this cycle $w_{T F 2}$ is as follows:

$$
\begin{aligned}
w_{T F 2} & =\frac{W_{T F 1}+W_{T F 2}}{l_{a 2} t} \\
& =\frac{W_{T F 1}+A_{L-c 2}-A_{U-c 2}}{l_{a 2} t}
\end{aligned}
$$

Specific total work of fracture (according to the description in Tables 3, 4, 5) can be calculated for a number of ligament lengths conveniently from a single cyclic test. Necessary calculations for specific work of fracture are shown in Tables 3, 4, 5 as an example. For LDPE, the total work of tear fracture in zone $\mathrm{I}\left(W_{T F-I}\right)$ was needed to be measured additionally from a separate tear test (Table 4). Noticeably, bending dissipation $\left(W_{d b}\right)$ accumulates in subsequent cycles. Extrapolation of specific total work of fracture along ligament length will result in specific essential work of tear fracture.

Specific essential work of fracture (SEWF) $\left(w_{T e}\right)$ for PET tear using zone I model and with no consideration of bending dissipation was calculated as 5.44 $\mathrm{N} / \mathrm{mm}$; when considering bending, it was calculated as $4.80 \mathrm{~N} / \mathrm{mm}$; having a difference of $11.6 \%$. For LDPE tear fracture with and without consideration of bending dissipation and using zone II model, SEWF were 11.84 $\mathrm{N} / \mathrm{mm}$ and $11.1 \mathrm{~N} / \mathrm{mm}$ respectively; this corresponds with a difference of $6.25 \%$. However, specific essential work of laminate tear fracture was $4.524 \mathrm{~N} / \mathrm{mm}$, which is strikingly low (Fig. 11). In the current case, SEWF for laminate appeared to not represent the laminate property correctly. A zone I model was used for this calculation. Further investigations could address this issue.

\section{Scanning electron microscopy}

In the post-test tearing specimens (in MD) three sections were cut perpendicularly to the initial crack at 1,7, and $13 \mathrm{~mm}$ away from the initial crack tip, as illustrated in Fig. 12b. The aim was to study the fracture surface,

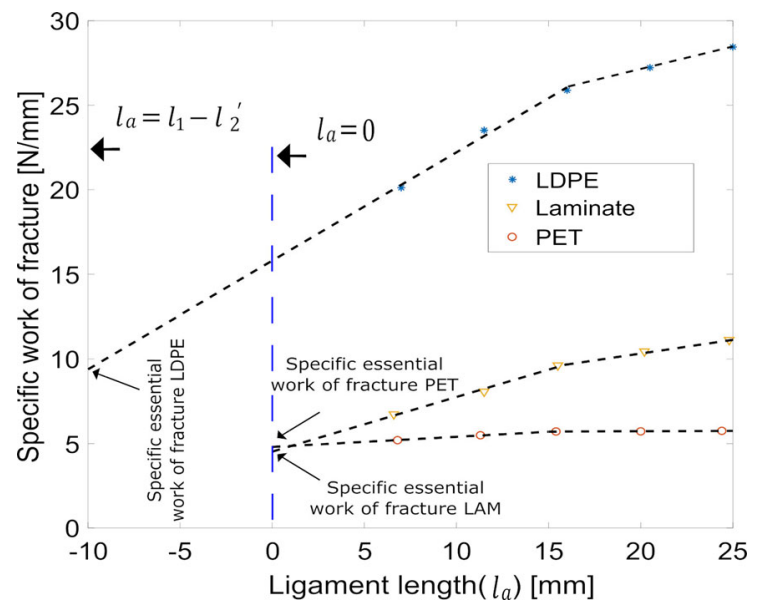

Fig. 11 Tearing specific work of fracture for different materials

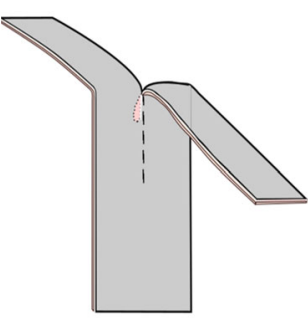

(a)

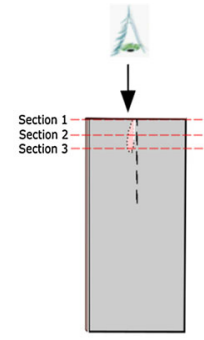

(b)

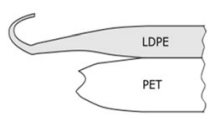

(c)
Fig. 12 Schematic of a Post-test trouser tear specimen of the laminate $\mathbf{b}$ section for crack tip SEM observation and $\mathbf{c}$ delamination and substrates' deformations

thinning of the materials prior to the failure and delamination in the laminate. The cross sections were cut with sharp scissors and were gold-coated in a Hitachi E-1030 Ion Sputter Coater. A Field Emission (SEM) Hitachi S4800 electron microscope was utilized for imaging.

The SEM image of a section (Sect. 1 according to Fig. 12b) near crack tip in Fig. 13a shows significant delamination due to tearing. Observations of PET fracture surface in Fig. 13b demonstrated that the nature of PET fracture is less ductile and that the plastic zone spreads less from the fracture surface. The thinning of PET prior to failure is also local to the fracture surface.

LDPE crack surface fracture appeared highly ductile (Fig. 13c); more importantly, there was a significant reduction in thickness which indicates damage on LDPE in the laminate is due to thinning.

Further observation on all three sections according to Fig. 12b agreed that the spread of this thickness reduction increases with tear crack propagation. An increase in the area that experiences reduction in thick- 


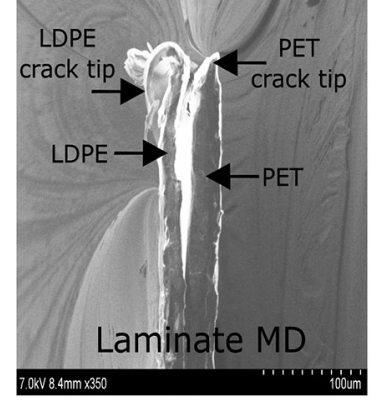

(a)

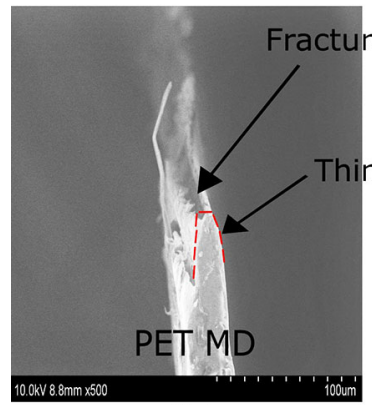

(b)

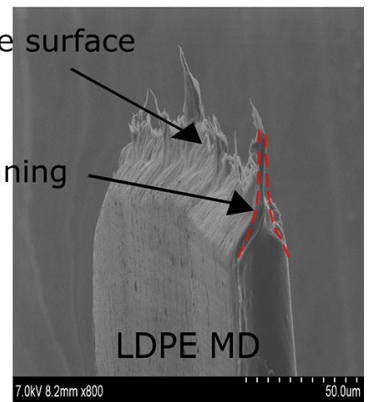

(c)
Fig. 13 SEM-micrographs of a crack tip and b, c fracture surface of LDPE-PET laminate in machine direction

ness with crack propagation is equivalent to an increase in ligament length width $(h)$ (Fig. 9a).

Figure 12c schematically and Fig. 13a in SEM crack tip section view of a torn laminate illustrates significant local strain with thickness reduction in the LDPE layer, relatively smaller thickness reduction, and strain of the PET layer and delamination of the interface. With increasingly torn ligament, more area is delaminated, and width of delamination is increased. As a result, more LDPE is unconstrained and therefore available for thinning (Fig. 2b).

\section{Results and discussion}

LDPE layer in the laminate is more ductile than PET layer. As a result, the spread of the plastic zone (Fig. 9b) is significantly larger than that of PET. In a laminate, the LDPE film is constrained by the interface, and the plastic zone cannot spread as in a stand-alone layer. This causes the laminate total work of fracture to be lower than that of individual layers combined (LDPE+PET in Fig. 14) for lower ligament length. However, as the crack propagates, delamination was observed to

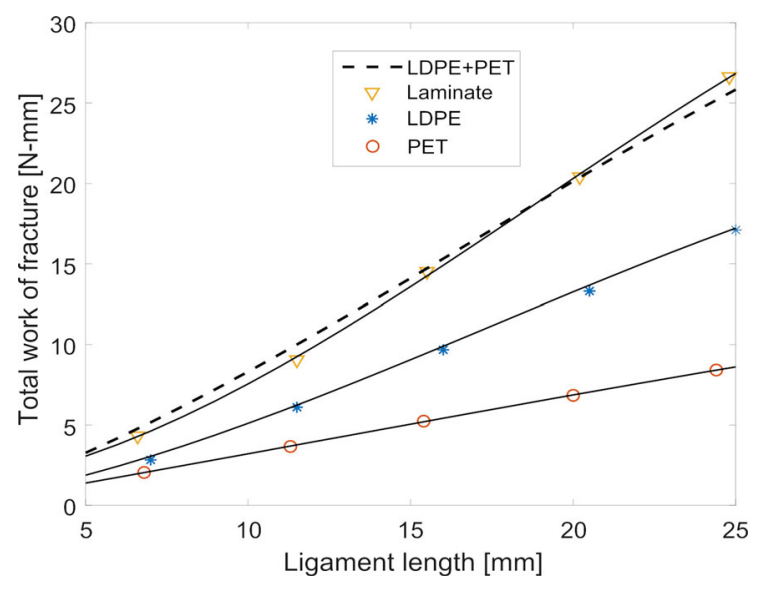

Fig. 14 Tearing total work of fracture for various materials

increase along the trouser-legs widths (Fig. 2b). This increases the volume of unconstrained LDPE that can undergo plastic deformation (thinning). Also, there is additional energy dissipation from delamination. As a result, the TWF of laminate is higher than PET and LDPE TWF combined at a larger ligament length (Fig. 14). So, material anisotropy causes tear crack deviation anisotropy (different tear deviation at different material orientation) which results in delamination anisotropy. This explains the reason for laminate being more anisotropic in tear compared to its constituents.

Plastic dissipations in the trouser-legs during tear are regarded as non-essential work of fracture. Measuring the dissipation from the leg bending curvature renders the calculated SEWF more independent of leg width. This method of measurement can be beneficial when it is practical to minimize the number of tests by not testing for multiple leg width to omit any width effect. The formulation provided in this study can be applied to both uniform and non-uniform curvature distribution.

SEWF of PET measured by the proposed cyclic tearing was comparable with results found in the literature. The calculated SEWF for $50 \mu \mathrm{m}$ PET was $4.80 \mathrm{~N} / \mathrm{mm}$ in this study and $6.35 \mathrm{~N} / \mathrm{mm}$ for $250 \mu \mathrm{m}$ PET in the literature (Kim and Karger-Kocsis 2004). The current value was smaller because the dissipation of bending was regarded as non-essential work of fracture. The literature reports that smaller tear SEWF for thinner PET is expected (Kim and Karger-Kocsis 2004). LDPE was divided into two zones based on visual inspection, and the proposed zone II method bypasses any necessary calculation for near crack tip plastic dissipation. This method is applicable to materials that develop long lig- 
aments with steadily increasing plastic zone width during tear. The authors did not find SEWF for LDPE tear reported in the literature. However, the specific essential work of fracture for blown LDPE film in mode I was reported to be approximately $16 \mathrm{~N} / \mathrm{mm}$ for $150 \mu \mathrm{m}$ thick LDPE film. The SEWF is more anisotropic for thinner LDPE, and it ranges between 9 and $43 \mathrm{~N} / \mathrm{mm}$ for $15 \mu \mathrm{m}$ thick LDPE (Rennert et al. 2013). Macroscopic crack tip observation of a tear test suggests that since LDPE is more ductile and flexible, it tends to shift mode, and load more in mode I; therefore, a relation can be expected with mode I SEWF. In this study, the calculated SEWF of tear for $25 \mu \mathrm{m}$ LDPE was $11.1 \mathrm{~N} / \mathrm{mm}$, which is comparable with the results reported earlier in the literature. The calculated SEWF of the laminate of LDPE-PET was significantly low and necessitates further investigation. Moreover, although the thickness can also exert a significant effect on SEWF value (Kim and Karger-Kocsis 2004), merely one particular thickness for each film was tested.

Material anisotropy of PET and LDPE affects trouser tear load response; therefore, this also affects EWF. The deviation in tear crack propagation is also related to the anisotropy. The tear crack deviation appears to correlate well with the weakest mode I fracture toughness direction of the material (Figs. 15a and 16). Details of the center crack panel test for mode I fracture toughness determination can be found in Appendix B. Constrained by LDPE, PET tear crack deflected less in laminate than as a single layer. The more the PET orientation aligns with $45^{\circ}$, the weaker the material becomes for cracking in mode I according to Fig. 16a and tear crack deviation reduces according to Fig. 15a. The tear crack tends to remain straighter close to $45^{\circ}$ orientation. For a tear orientation that differs from $45^{\circ}$, the crack changes direction toward the left or right (approaching from MD or CD) to a certain degree until a saturation is reached. This relation between mode I and tearing was expected; as with crack propagation, tear becomes a mixture of mode I and mode III fracture. Noticeably, in a MD tear specimen, the mode I loading is in $\mathrm{CD}$; this $90^{\circ}$ shift holds for all other directions. The amount of delamination is positively correlated with the deviation of the crack in a laminate (Fig. 15a, b) meaning smaller delamination in the laminate for smaller crack deviation.

The tear tests were performed such that trouser-legs were separated vertically, which caused the tail of the tear specimen to hang and bend down due to gravity

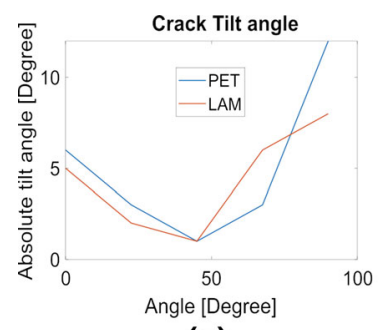

(a)

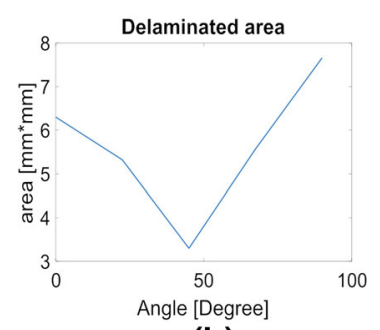

(b)
Fig. 15 Anisotropy during tearing a crack deviation in PET and laminate and $\mathbf{b}$ delamination in laminate. (For explanations of the colours in these figures, please refer to the web version of this article)

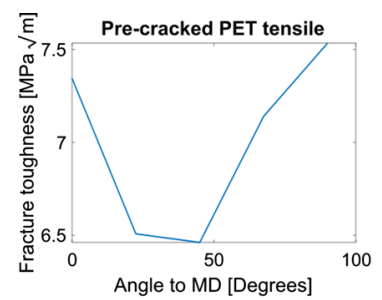

(a)

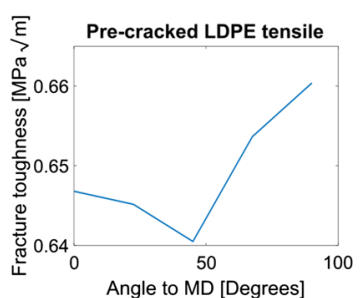

(b)

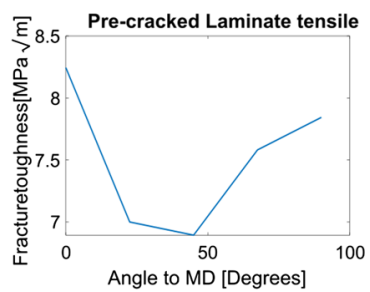

(c)

Fig. 16 Mode I fracture toughness of the tested materials at different orientations a PET, b LDPE and $\mathbf{c}$ laminate. (For explanations of the colours in these figures, please refer to the web version of this article)

(Fig. 2a). This can cause the bending curvature at the bottom leg to be larger than the top one and contribute to additional tear crack deviation. Particularly in the laminate, if the more compliant LDPE side is facing upwards, delamination width increases faster with tearing relative to when the PET side faces upwards. This can affect the SEWF and worth further investigation. This effect can be negated by pulling the tear specimen sidewise such that the tail hangs vertically.

\section{Conclusions}

Trouser tear test of PET, LDPE films, and the corresponding laminate have been examined in this study 
in five different material orientations. Propagation of tearing in these thermoplastics demonstrated deviation from the initial and parallel crack path with a mixed mode I and mode III. This was determined to be caused by the material anisotropy, and the deviation can be related to the difference in mode I fracture toughness at different material orientations. The crack tends to deflect toward the weakest material orientation. The amount of delamination was also discovered to be influenced by the material orientation.

The proposed cyclic tear test method for SEWF measurement could produce results comparable to those reported in the literature. Energy dissipation due to non-uniform bending of the trouser-legs was demonstrated to be significant in the tearing SEWF calculation and was therefore considered in this study. Analytical expressions for the calculation of non-uniform bending energy dissipation for a bi-linear isotropic hardening material model were presented. A variation of a twozone tear model was proposed to bypass any plastic dissipation calculation for SEWF calculation in LDPE. To measure the SEWF of laminates, delamination energy dissipation was accounted for. However, delamination appeared to expose more unconstrained LDPE that effects the laminate behaviour more than that caused by energy dissipation due to delamination. Further study is necessary to use EWF for characterization of laminate tear fracture. Also, this study particularly focused on thin LDPE, PET and their laminate. Additional studies are necessary to check the applicability of the presented methods and formulations for other polymers.

Acknowledgements Open access funding provided by Blekinge Institute of Technology. This work is a part of the research activity of the Model Driven Development and Decision Support project (MD3S) at Blekinge Institute of Technology, which is funded by the Swedish Knowledge and Competence Development Foundation (KKS). Special thanks to SEM laboratory at Shanghai Polytechnic University, China for allowing us to use their facility, and to Dr. Lun Zhao from Kunming University of Science and Technology in China for helping us with the SEM study.

Open Access This article is distributed under the terms of the Creative Commons Attribution 4.0 International License (http://creativecommons.org/licenses/by/4.0/), which permits unrestricted use, distribution, and reproduction in any medium, provided you give appropriate credit to the original author(s) and the source, provide a link to the Creative Commons license, and indicate if changes were made.

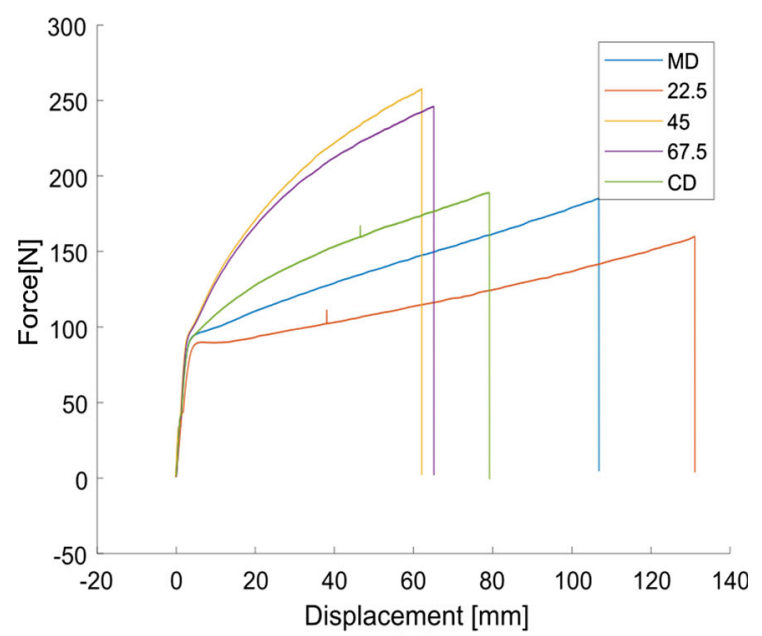

(a)

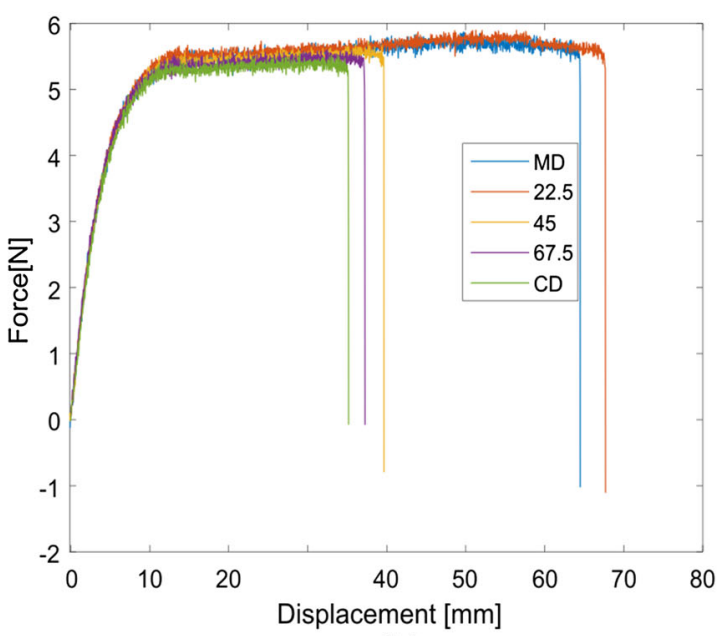

(b)

Fig. 17 Mechanical tensile test of a PET film and b LDPE film. (For explanations of the meanings of the colours in these figure legends, please refer to the web version of this article)

\section{Appendix A: Tensile test results}

The tensile tests were performed according to the standard ISO 527-3 (Standard 2018). Specimen length between the tensile grips was $100 \mathrm{~mm}$, width was 25 $\mathrm{mm}$ (Fig. 1b) and test speed was $20 \mathrm{~mm} / \mathrm{min}$. Representative force vs. displacement results, presented in Fig. 17a, indicate significant anisotropy both in maximum displacement and peak force for PET. The deviation in peak force for LDPE tensile test responses was relatively small, but material oriented close to MD was determined to withstand higher strain (Fig. 17b). 


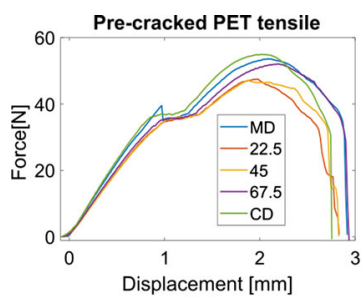

(a)

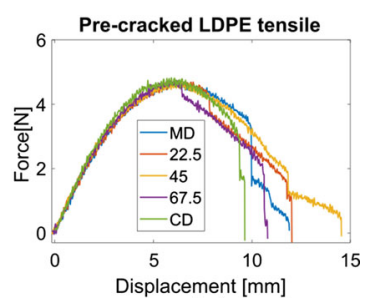

(b)

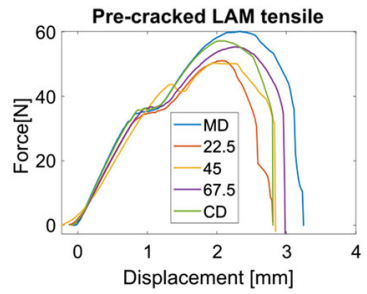

(c)

Fig. 18 Center crack test results at different material orientations. a PET F-D response, b LDPE F-D response and $\mathbf{c}$ laminate $\mathrm{F}-\mathrm{D}$ response. (For explanations of the colours in these figure legends, please refer to the web version of this article)

Table 6 Peak force at different material orientation of the tested materials

\begin{tabular}{|c|c|c|c|c|c|}
\hline & $M D$ & $22.5^{\circ}$ & $45^{\circ}$ & $67.5^{\circ}$ & $C D$ \\
\hline Peak load-LDPE (N) & 4.72 & 4.70 & 4.47 & 4.77 & 4.38 \\
\hline Peak load-PET (N) & 53.60 & 47.49 & 47.15 & 52.10 & 54.98 \\
\hline Peak load-Laminate $(\mathrm{N})$ & 60.16 & 51.08 & 50.30 & 55.32 & 57.23 \\
\hline
\end{tabular}

\section{Appendix B: Center crack (CC) panel test}

The center crack specimen dimension used in an early study was adopted as a reference (Kao-Walter 2004). However, because of the dimension of the produced LDPE-PET laminate, the CC specimens were downscaled by a factor of 2.3 to fit the material width in this specific case. Finally, $100 \mathrm{~mm}$-long and $41 \mathrm{~mm}$-wide CC specimens of LDPE, PET, and their laminate were tested with a center crack of $20 \mathrm{~mm}$ (Fig. 1b). The crack preparation technique affects the fracture property significantly (Martínez et al. 2010); since this effect was inevitable, it was ensured that the specimens preparation conditions were repeatable. Figure 18 presents the force displacement $(\mathrm{F}-\mathrm{D})$ responses of these tests, and Table 6 displays the peak forces.

\section{Appendix C: Peel test}

The adhesion between PET and LDPE can be quantified based on a peel test. Fracture energy of delamination was determined using Kinloch's theoretical model of peel (Kinloch et al. 1994). $50 \mathrm{~mm}$-wide peel specimens were used, and LDPE film was peeled off at $90^{\circ}$ and $180^{\circ}$ until a steady force response had been achieved. Steady force of peeling was the only quantity used from the peel tests. Necessary tensile properties of LDPE were also calculated based on the tensile test performed in this study. Fracture energy of delamination from peel was calculated to be $33 \mathrm{~J} / \mathrm{m}^{2}$.

\section{References}

Andreasson E, Mehmood N, Kao-Walter S (2013) Trouser tear tests of two thin polymer films. In: 13th international conference on fracture, ICF13

Andreasson E, Kao-Walter S, Ståhle P (2014) Micro-mechanisms of a laminated packaging material during fracture. Eng Fract Mech 127:313-326

Bárány T, Ronkay F, Karger-Kocsis J, Czigány T (2005) In-plane and out-of-plane fracture toughness of physically aged polyesters as assessed by the essential work of fracture (ewf) method. Int J Fract 135(1-4):251-265

Bjerkén C, Kao-Walter S, Stahle P (2006) Fracture mechanisms of a thin elastic plastic laminate. In: Gdoutos EE (ed) Fracture of nano and engineering materials and structures. Springer, Berlin, pp 927-928

Islam MS, Zhang D, Mehmood N, Kao-Walter S (2016) Study of shear dominant delamination in thin brittle-high ductile interface. Proc Struct Integr 2:152-157

JA H (2010) Plot digitizer. Sourceforge. Net. http://plotdigitizer. sourceforge.net/. Accessed 3 June 2018

Kao-Walter S (2004) On the fracture of thin laminates. PhD thesis, Blekinge Institute of Technology

Kao-Walter S, Ståhle P, Chen SH (2006) A finite element analysis of a crack penetrating or deflecting into an interface in a thin laminate. Key Eng Mater Trans Tech Publ 312:173-178

Kao-Walter S, Hu M, Walter M, Leon A (2009) A comparison of 2-zone and 3-zone models in tearing based on essential work of fracture. In: 12th Int. conference on fracture, Natural resources Canada

Kao-Walter S, Walter M, Dasari A, Leon A (2011) Tearing and delaminating of a polymer laminate. Key Eng Mater Trans Tech Publ 465:169-174

Kim HS, Karger-Kocsis J (2004) Tearing resistance of some co-polyester sheets. Acta Mater 52(10):3123-3133

Kinloch A, Lau C, Williams J (1994) The peeling of flexible laminates. Int J Fract 66(1):45-70

Mai Y, Cotterell B (1984) The essential work of fracture for tearing of ductile metals. Int J Fract 24(3):229-236

Martínez A, Segovia A, Gamez-Perez J, Maspoch ML (2010) Essential work of fracture analysis of the tearing of a ductile polymer film. Eng Fract Mech 77(14):2654-2661 
Rennert M, Nase M, Lach R, Reincke K, Arndt S, Androsch R, Grellmann W (2013) Influence of low-density polyethylene blown film thickness on the mechanical properties and fracture toughness. J Plast Film Sheet 29(4):327-346

Rivlin R, Thomas AG (1953) Rupture of rubber. i. characteristic energy for tearing. J Polym Sci 10(3):291-318

Standard A (1993) Standard test method for tear-propagation resistance of plastic film and thin sheeting by a single-tear method. ASTM International, West Conshohocken, PA. https://www.astm.org

Standard I (2018) Plastics-Determination of tensile propertiespart 3: test conditions for films and sheets. ISO

Wong JS, Ferrer-Balas D, Li RK, Mai YW, Maspoch ML, Sue HJ (2003) On tearing of ductile polymer films using the essential work of fracture (ewf) method. Acta Mater 51(16):4929-4938
Zhang DF, Islam S, Andreasson E, Kao-Walter S (2016) Modeling and study of fracture and delamination in a packaging laminate. In: 3rd international conference on material engineering and application (ICMEA), NOV 12-13, 2016. Peoples R China, Atlantis Press, Shanghai, pp 408-414

Publisher's Note Springer Nature remains neutral with regard to jurisdictional claims in published maps and institutional affiliations. 